\title{
Use of 3D Printing in Preoperative Planning and Training for Aortic Endovascular Repair and Aortic Valve Disease
}

\author{
Eduardo Nascimento Gomes ${ }^{1}, \mathrm{MD}$; Ricardo Ribeiro Dias' ${ }^{1}$ MD, PhD; Bruno Aragão Rocha², MD; José Augusto Duncan \\ Santiago' , MD; Fabrício José de Souza Dinato' ', MD; Eduardo Keller Saadi ${ }^{3}$, MD, PhD; Walter J. Gomes ${ }^{4}$, MD, PhD; Fabio \\ B. Jatene', MD, PhD
}

\begin{abstract}
Introduction: Three-dimensional (3D) printing has become an affordable tool for assisting heart surgeons in the aorta endovascular field, both in surgical planning, education and training of residents and students. This technique permits the construction of physical prototypes from conventional medical images by converting the anatomical information into computer aided design (CAD) files.

Objective: To present the 3D printing feature on developing prototypes leading to improved aortic endovascular surgical planning, as well as transcatheter aortic valve implantation, and mainly enabling training of the surgical procedure to be performed on patient's specific condition.

Methods: Six 3D printed real scale prototypes were built representing different aortic diseases, taken from real patients,
\end{abstract}

to simulate the correction of the disease with endoprosthesis deployment.

Results: In the hybrid room, the 3D prototypes were examined under fluoroscopy, making it possible to obtain images that clearly delimited the walls of the aorta and its details. The endovascular simulation was then able to be performed, by correctly positioning the endoprosthesis, followed by its deployment.

Conclusion: The 3D printing allowed the construction of aortic diseases realistic prototypes, offering a 3D view from the twodimensional image of computed tomography (CT) angiography, allowing better surgical planning and surgeon training in the specific case beforehand.

Keywords: Aorta/Surgery. Aorta, Thoracic. Endovascular Procedures. Aneurysm. Aneurysm, Dissecting. Imaging, ThreeDimensional. Models, Cardiovascular.

\begin{tabular}{llll}
\hline \multicolumn{2}{l}{ Abbreviations, acronyms \& symbols } \\
\hline 3D & = Three-dimensional & NYHA & $=$ New York Heart Association \\
An & $=$ Aneurysm & PAU & $=$ Penetrating aortic ulcer \\
CAD & $=$ Computer aided design & PLA & $=$ Polylactic acid \\
CT & $=$ Computed tomography & STL & $=$ STereoLithography \\
DICOM & = Digital Imaging and Communications in Medicine & TL & $=$ True lumen \\
FDM & $=$ Fused deposition modeling & UV & $=$ Ultraviolet \\
FL & $=$ False lumen & &
\end{tabular}

${ }^{1}$ Cardiovascular Surgery Division, Instituto do Coração do Hospital das Clínicas da Faculdade de Medicina da Universidade de São Paulo (InCor-HCFMUSP), São Paulo, SP, Brazil.

${ }^{2}$ Instituto de Radiologia do Hospital das Clínicas da Faculdade de Medicina da Universidade de São Paulo (InRad-HCFMUSP), São Paulo, SP, Brazil.

${ }^{3}$ Department of Cardiovascular Surgery, Hospital de Clínicas de Porto Alegre (HCPA), Universidade Federal do Rio Grande do Sul (UFRGS), Porto Alegre, RS, Brazil. ${ }^{4}$ Cardiology and Cardiovascular Surgery Disciplines, Escola Paulista de Medicina da Universidade Federal de São Paulo (EPM-UNIFESP), São Paulo, SP, Brazil.

This study was carried out at the Cardiovascular Surgery Division, Instituto do Coração do Hospital das Clínicas da Faculdade de Medicina da Universidade de São Paulo (InCor-HCFMUSP), São Paulo, SP, Brazil.
No financial support.

No conflict of interest.

Correspondence Address:

Eduardo Nascimento Gomes

Instituto do Coração do Hospital das Clínicas da Faculdade de Medicina da Universidade de São Paulo (InCor-HCFMUSP)

Av. Dr. Enéas Carvalho de Aguiar, 44 - Cerqueira César - São Paulo, SP, Brazil

Zip code: 05403-900

E-mail: eduardongomes85@gmail.com

Article received on May $21^{\text {th }}, 2018$. Article accepted on August 16 ${ }^{\text {th }}, 2018$. 


\section{INTRODUCTION}

In recent decades, the endovascular treatment of aortic diseases, such as aneurysms, dissections, and penetrating ulcers, became widely used for being a minimally invasive procedure with satisfactory long-term outcomes, acceptable mortality rates, and lower postoperative complications ${ }^{[1-6]}$.

For assisting heart surgeons in the aorta endovascular field, three-dimensional (3D) printing (or rapid prototyping) has become an affordable reality and it is rapidly expanding its applications, both in surgical planning and in education and training of residents and students $s^{[7-14]}$.

This technique permits the construction of physical prototypes from conventional medical images, such as computed tomography (CT) scan and magnetic resonance imaging, by converting the anatomical information into computer aided design (CAD) files ${ }^{[11-13]}$.

This process is performed by using a specific software and requires both the anatomical knowledge for selecting the regions of interest and the acquaintance with graphic features to perform the steps.

Therefore, our objective is to present the 3D printing feature on developing prototypes leading to improved aortic endovascular surgical planning, as well as transcatheter aortic valve implantation, and mainly enabling training of the surgical procedure to be performed on patient's specific condition.

\section{METHODS}

From multi-institutional image database, six 3D real scale prototypes were built representing different aortic diseases, taken from real patients, to simulate the correction of the disease with endoprosthesis deployment in the hybrid room.

For the prototype assembly, the image files used were Digital Imaging and Communications in Medicine (DICOM $\left.{ }^{\oplus}\right)$ CT angiographies obtained in 64-slice CT scanners, using a mechanical injector pump with $4 \mathrm{~mL} / \mathrm{s}$ contrast infusion speed and isotropic voxels at $0.625 \mathrm{~mm}$.

These $\mathrm{DICOM}^{\oplus}$ files were exported to the software Invesalius ${ }^{\oplus}$ (Centro de Tecnologia da Informação Renato Archer, Campinas, Brazil), to target the aortic lumen and its main branches and create a 3D model of the aortic lumen in STereoLithography (STL)

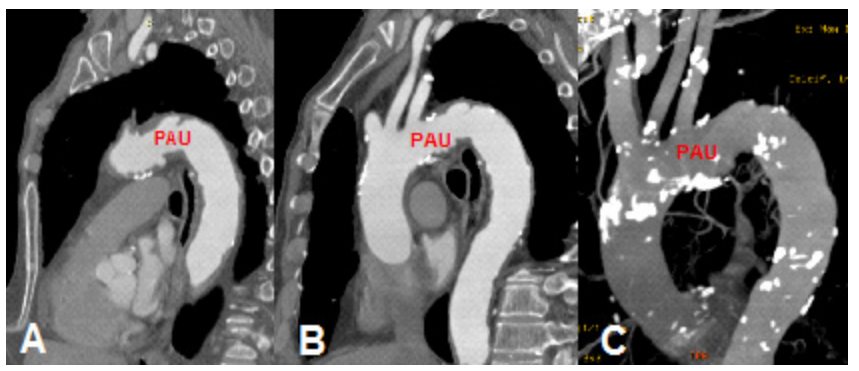

Fig. 1 - Case 1: computed tomography (CT) angiography of penetrating aortic ulcer (PAU) on the thoracic aorta with a maximal diameter of $57 \mathrm{~mm}$. A) Oblique sagittal section on maximal diameter. B) Oblique sagittal section showing its relation to the great vessels. C) Reconstruction of the aneurysm. format, which is compatible with 3D printing. Then, these files were exported to Fusion $360^{\circledR}$ (Autodesk Inc.), a CAD manipulation software, to extrude a $2 \mathrm{~mm}$ uniform artificial wall, obtaining a hollow file representing the aortic lumen, the luminogram.

Next, these files were sent to 3D printers. In this study, we chose to use two different printing technologies: a fused deposition modeling (FDM) technology printer, the MakerBot Replicator $2^{\oplus}$, which uses a temperature extruder nozzle for a plastic filament called polylactic acid (PLA) in red color; and a multijet technology printer, the 3D Systems ProJet $3510^{\oplus}$, which employs an ultraviolet (UV) photocurable resin, allowing printing on a semitransparent material.

Finally, the printed 3D models were taken to the hybrid operating room and examined under fluoroscopy, making it possible to obtain images that clearly delimited the walls of the aorta and its details. The endovascular simulation was then able to be performed, by correctly positioning the endoprosthesis followed by its deployment.

\section{Clinical}

Case 1: 65-year-old male, with a penetrating aortic ulcer (PAU) on the descending thoracic aorta. On the CT angiography (Figure 1), it seemed to start right after the origin of the left subclavian artery, extending to the descending thoracic aorta, with a maximum diameter of $57 \mathrm{~mm}$.

Patient was selected for endovascular treatment. The initial planning suggested that the endoprosthesis could be anchored in Zone $3^{[15]}$ with the free flow zone over the left subclavian artery.

Case 2: 67-year-old male, with a descending aorta aneurysm and distal involvement of the aortic arch with a maximum diameter of $10.0 \times 9.2 \mathrm{~cm}$ (Figure 2).

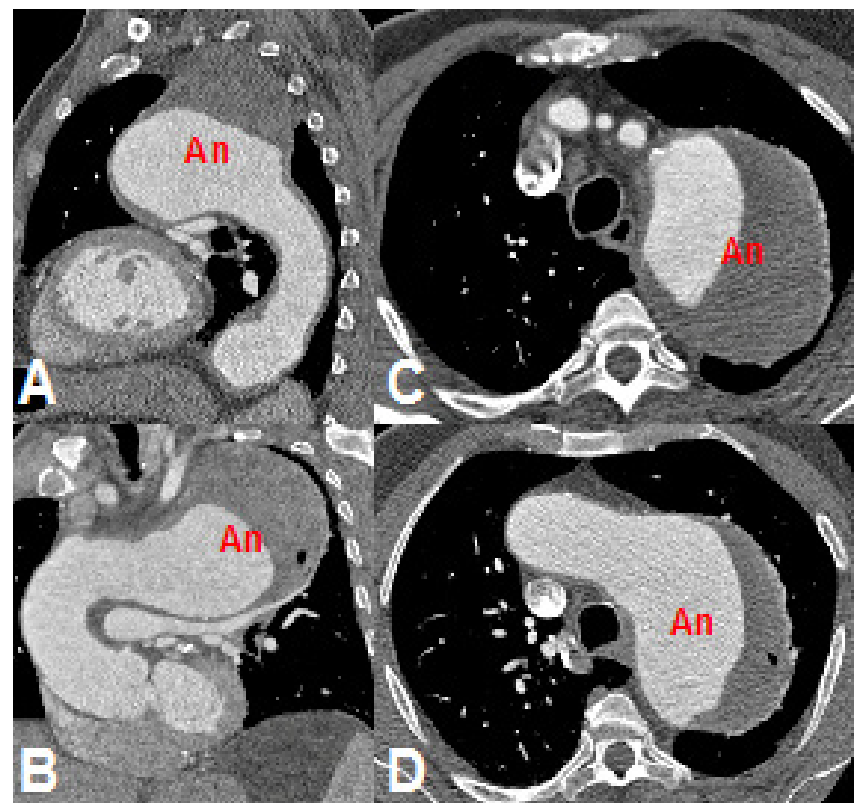

Fig. 2 - Computed tomography (CT) angiography of the $10 \mathrm{~cm}$ thoracic aortic aneurysm (An). A) Sagittal section. B) Oblique sagittal section at maximum diameter. C) Axial section at the level of the great vessels. D) Axial section at the level of the aortic arch. 


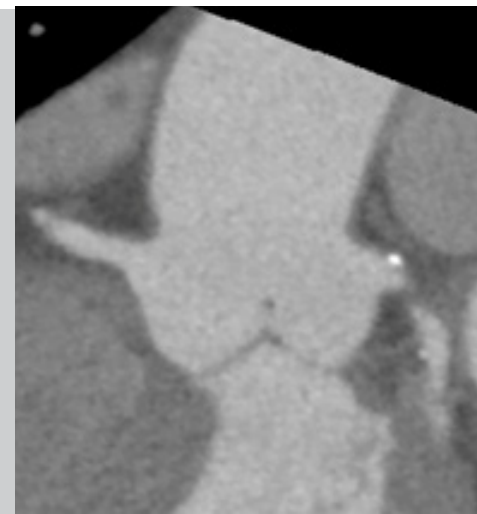

Fig. 3 - Case 3: computed tomography (CT) angiography identifying the anatomical features of the left ventricle outflow tract.

Case 3: 75-year-old male, being followed-up by the Cardiology team for severe aortic valve stenosis with an ejection fraction of 30\% in New York Heart Association (NYHA) functional class III (Figure 3).

Case 4: 68-year-old male, with asymptomatic infrarenal abdominal aortic aneurysm extending to the iliac arteries with maximum diameter of $67 \mathrm{~mm}$ (Figure 4).

Case 5: 64-year-old male, 3 years follow-up for Stanford type B chronic aortic dissection, with acute abdominal pain, undergoing surgical treatment (Figure 5).

Case 6: 67-year-old female, Stanford type A chronic dissection, with acute dyspnea and chest pain undergoing ascending aorta replacement (Figure 6).

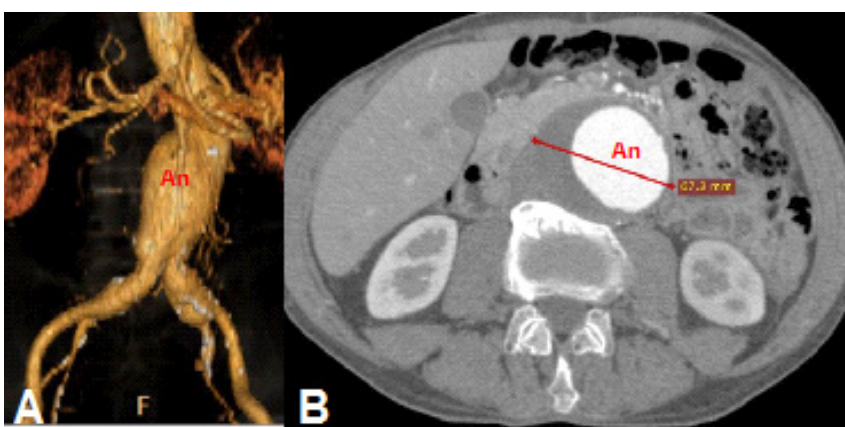

Fig. 4-Case 4: computed tomography (CT) angiography of abdomina aortic aneurysm (An). A) Digital reconstruction of the aneurysm. B) Axial section measuring the aneurys, $67.2 \mathrm{~mm}$ in diameter.

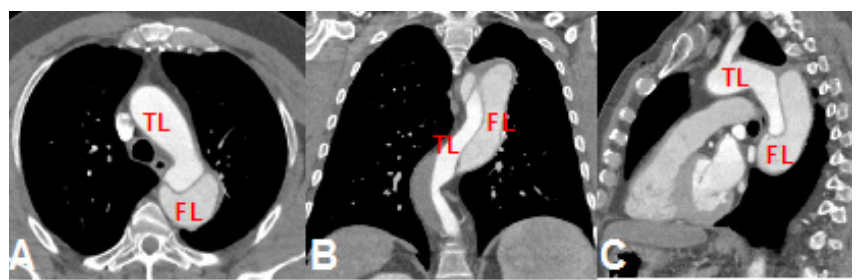

Fig. 5 - Case 5: computed tomography (CT) angiography of aortic dissection showing true lumen (TL) and false lumen (FL). A) Axial section on the level of the arch. B) Coronal section of the descending thoracic aorta. C) Sagittal section of the aortic arch.

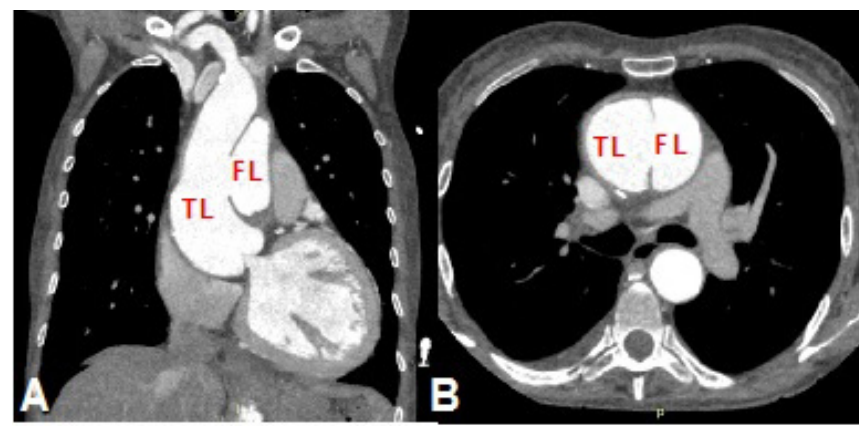

Fig. 6 - Case 6: computed tomography (CT) angiography of aortic dissection showing true lumen ( $T L$ ) and false lumen (FL). A) Coronal section showing the dissection in the ascending aorta with its intimal tear. B) Axial section at the level of the intimal tear.

\section{RESULTS}

Using 3D printing, a specific full-scale endoluminal anatomy prototype (luminogram) was obtained in detail in all cases, allowing a proper surgical technique planning, with perfect 3D understandable aorta visualization and revalidation of the endovascular planning.

In case 1, the prototype showed a difficult proximal landing zone at Zone $2^{[15]}$ (Figure 7). During the procedure, the endoprosthesis backed off and anchored itself inside the ulcer, requiring an extension anchored in Zone $2^{[15]}$ with free flow over the left carotid artery for proper treatment, completely covering the penetrating ulcer (Figure 8).

In case 2, the prototype (Figures 9 and 10; Video 1) showed the need for an additional procedure to create a proximal landing zone in Zone $1^{[15]}$, either by exclusive endovascular technique, such as the chimney, or by hybrid techniques with extra-anatomical tube or debranching ${ }^{[16-19]}$ (Figure 11; Video 2).

In case 3, the left ventricular outflow tract was well represented, making it possible to estimate the size of the aortic annulus and the height of the coronary ostia, although it was not possible to reconstruct the aortic cusps. However, this reconstruction limitation did not prevent the prototype's use for preoperative training (Figure 12).

In case 4, the abdominal aortic aneurysm 3D prototype (Figure 13) reinforced that the proximal landing zone was a difficult one but it was adequate to release aortic bi-iliac endoprosthesis, due to its angle (Video 3).

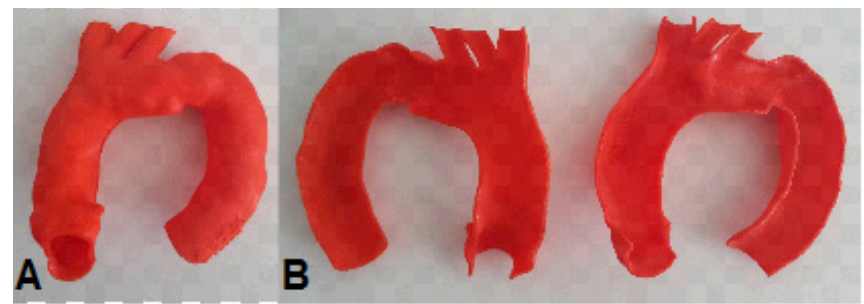

Fig. 7 - Case 1: prototype printed on red polylactic acid (PLA). A) View from the front, showing the penetrating aortic ulcer in the arch. B) Prototype split, showing the inside view. 


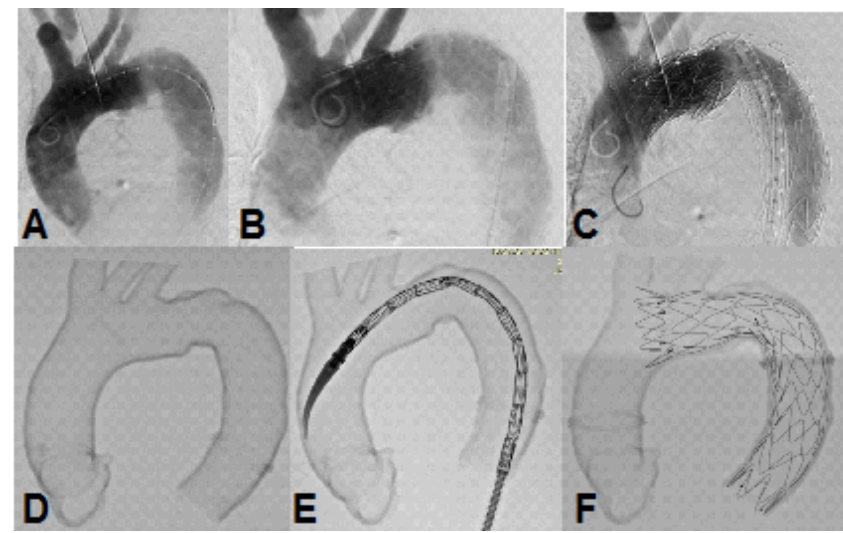

Fig. 8 - Case 1: A) Fluoroscopy of the patient. B) Failure after deploying first endoprosthesis. C) After second endoprosthesis deployment on Zone 2. D) Fluoroscopy of the prototype. E) Simulation of endoprosthesis deployment on prototype. F) Prototype after endoprosthesis deployment.

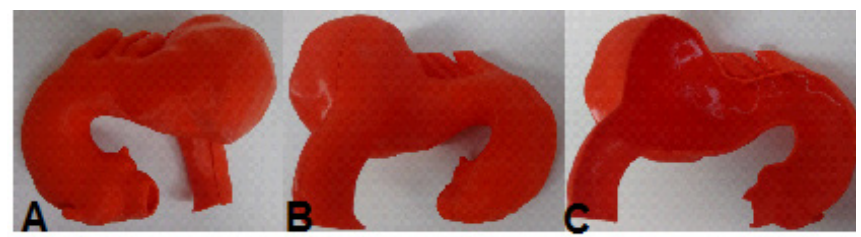

Fig. 9 - Case 2: prototype printed on red polylactic acid (PLA). A) Frontal view of the prototype. B) Rear view. C) Inside view.

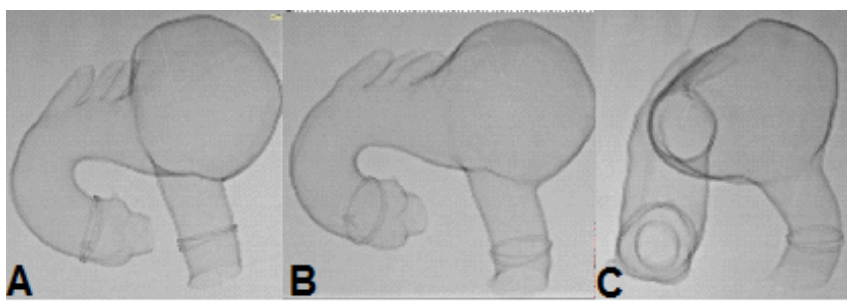

Fig. 10 - Case 2: fluoroscopy of the prototype. A) On zero degrees. B) $30^{\circ}$ left. C) $60^{\circ}$ left.

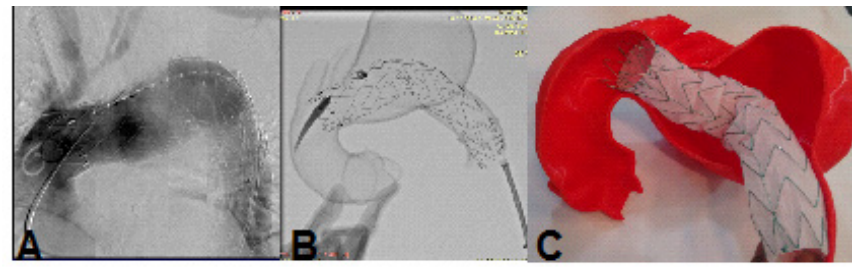

Fig. 11 - Case 2: comparison between the prototype and the procedure. A) Fluoroscopy of the patient after procedure. B) Fluoroscopy of the prototype after procedure. C) Endoprosthesis inside prototype after deployment.

In cases 5 (Figure 14) and 6 (Figure 15), the aortic dissection was well represented, the intimal tear was spotted, and the length of the false lumen was sized. In the type A dissection, the prototype allowed the evaluation of a possible unconventional

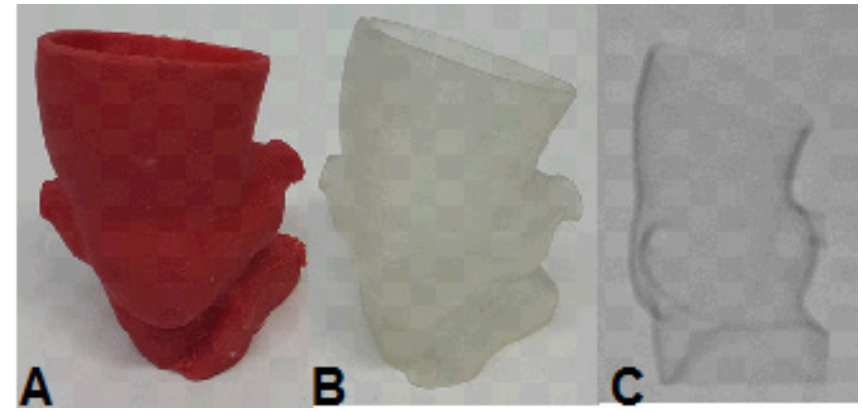

Fig. 12 - Case 3: prototype. A) Printed on red polylactic acid (PLA). B) Printed on semitransparent resin. C) Fluoroscopy of the prototype.

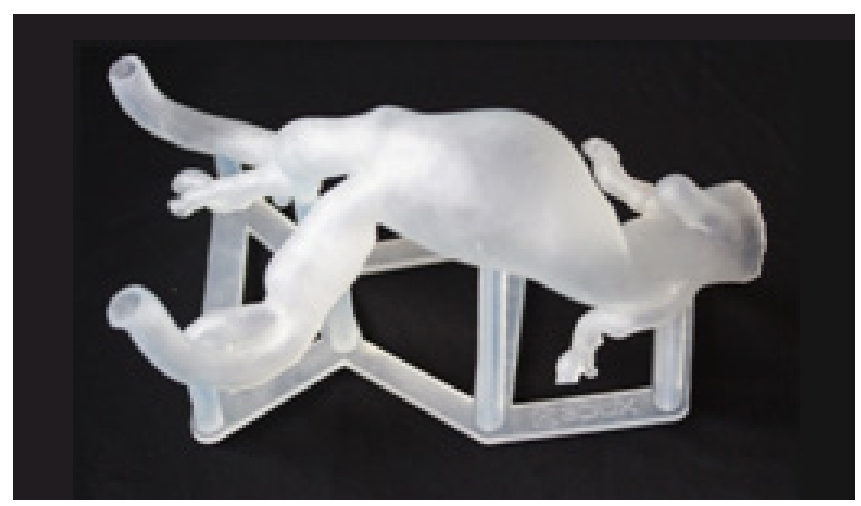

Fig. 13 - Case 4: prototype printed on semitransparent resin.

Video 1 - Case 2: fluoroscopy of the prototype.

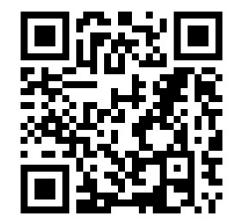

Video 2 - Case 2: simulation of endoprosthesis deployment on prototype.

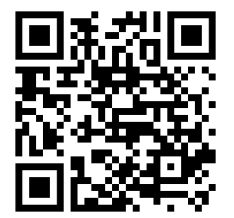

Video 3 - Case 4: fluoroscopy of the prototype.

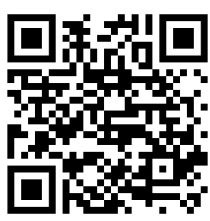

treatment for this segment of the compromised aorta or an endovascular treatment with customized stent. In type B dissection, the prototype served as a good model for training the identification of true and false lumens (Figure 16). 


\section{DISCUSSION}

From our experience, the prototypes used proved to be efficient and reliable for understanding and possibly refining the operative strategy in the assessed aortic diseases.

In recent years, 3D printing technology proved to be a very useful tool in different fields of medicine. Among the various medical applications, orthopedic surgery, otolaryngology, neurosurgery, and plastic and maxillofacial surgeries are the most benefited by the method ${ }^{[20,21]}$. In part, this is due to lower

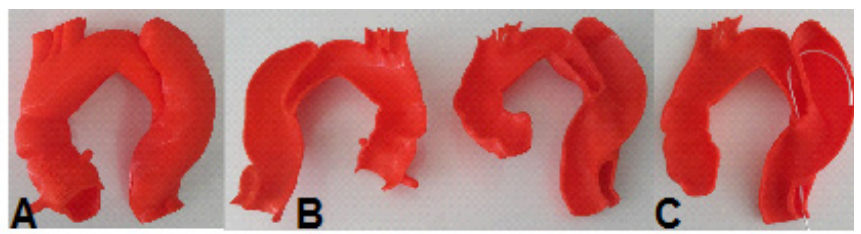

Fig. 14 - Case 5: Prototype printed on red polylactic acid (PLA). A) Frontal view showing the false lumen. B) Prototype split, inside view showing the dissection. C) Prototype split with wire coming distally from true lumen to false lumen through the intimal tear.

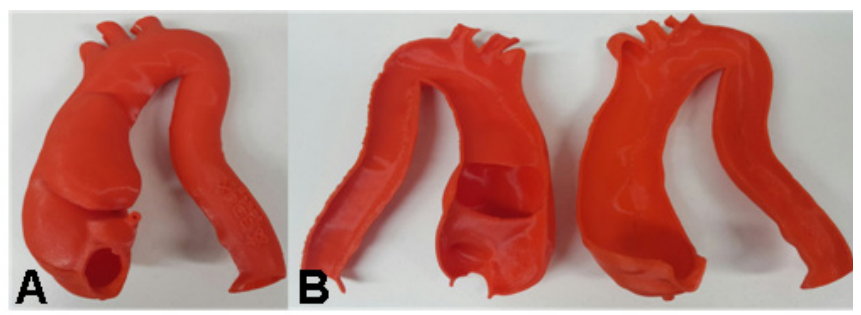

Fig. 15 - Case 6: Prototype printed on red polylactic acid (PLA). A) View from the front showing the extent of the false lumen. B) Prototype split showing the intimal tear.

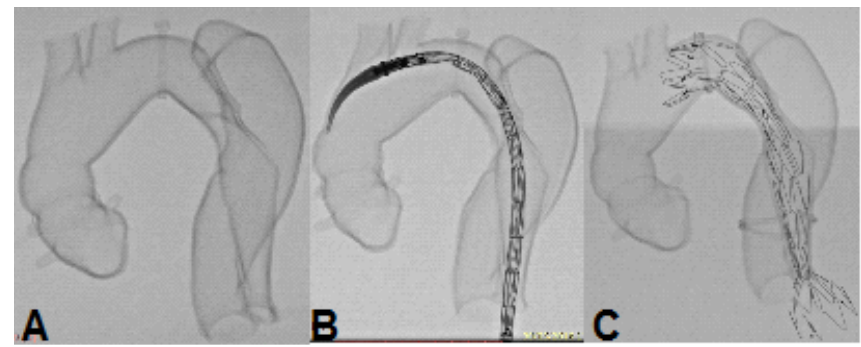

Fig. 16 - Case 5: simulation of endoprosthesis deployment on prototype. A) Fluoroscopy of the prototype. B) Simulation of endoprosthesis deployment on prototype. C) Prototype after endoprosthesis deployment.

technical complexity in extracting the CAD models from bone structure images, owing to its marked contrast between its limits with adjacent soft tissue structures. Also, because there are many diseases to which the 3D vision and its understanding become extremely essential for a successful surgical treatment.

Recently, a growing number of studies on 3D printing applications have been emerging, enhancing understanding and surgical planning of cardiothoracic diseases, mainly because of technical development and popularization of imaging methods such as CT angiography and cardiac magnetic resonance imaging $^{[11-13]}$.

One of the 3D printing limitations in cardiothoracic diseases is the possibility of errors during the segmentation from CT angiography images to obtain the STL file. This segmentation process is observerdependent and it requires an extensive anatomical knowledge so that, during these steps, it is guaranteed that all tissues relevant to the case are selected and regions outside the organs of interest and not real structures, such as artifacts, are not represented.

In addition, many scans have satisfactory imaging from a diagnostic point of view, but they are full of shadows and artifacts, preventing the extraction process of the regions of interest in an appropriate manner.

In this study, the main use of 3D printing was to better preoperatively understand the patient's anatomy, especially to accurately comprehend the dimensional scale, angulations, and other elements, as the aneurysmal landing zones and the location of a penetrating ulcer or intimal tear.

A cost-effectiveness assessment of prototyping was not carried out in this study, however, due to its affordable cost, it may prove to be cost effective. First, for allowing to better define the type, length, and oversizing of the endoprosthesis, reducing the chance for further extension. Second, it allows the surgeon to practice the surgery before the actual operation. Thus, at the time of the procedure, the time saving is also considerable due to the high cost of a hybrid room; the reduced use of radiopaque contrast decreases the costs and the insult to renal function, especially in patients with kidney failure; and reducing the use of radiation, the exposure of the patient and staff to its hazardous effect is shortened. And third, the reproduction of knowledge through educational activities or training of other surgeons, residents, and medical students using prototype simulators, which can be used in the hybrid room, can have an impact on reducing the technical learning curve ${ }^{[22]}$.

Particularly, the conditions affecting the aortic arch are more challenging, because of the concern of cerebral protection in case of an open surgery or of maintaining cerebral perfusion by endovascular techniques or a hybrid approach. These conditions can be classified as proposed by Mitchell and Ishimaru ${ }^{[15-19]}$ according to the proximal landing zone of the endoprosthesis. Zones 3 and 4 are routinely treated by endovascular techniques; and Zones 0, 1, and 2 require a more complex approach, either endovascular, hybrid, or conventional. In our service, for landing in Zone 1 or 2, we use the hybrid technique with revascularization of the great vessels (debranching) in order to create a favorable landing zone to deploy the endoprosthesis without compromising the brain and the left upper limb perfusion.

The evaluation of the 3D model may show a more proximal landing zone than predicted by the analysis of $\mathrm{CT}$, as occurred in the cases 1 and 2, requiring a change in the operating strategy, such as an initial hybrid approach (case 2) or the sacrifice of the left subclavian artery (case 1), saving an endoprosthesis extension ${ }^{[16-19]}$.

With these case series, we add more examples of the feasibility of using 3D printing in cardiothoracic surgery, reinforcing its potential benefits in understanding, surgical planning, and also in training and education. In fact, this study is an initial experience to establish a closer contact with prototyping, however, more work is needed in this field to objectively assess 
the cost-effectiveness, by understanding the case studied, and the possible clinical implications, such as saving endoprosthesis extensions and the possibility of surgical time reduction, along with decreased anesthetic time, contrast, and radiation doses or surgical outcome improvement.

\section{CONCLUSION}

The 3D printing allowed the construction of aortic diseases realistic prototypes, offering a 3D view from the two-dimensional image of CT angiography, allowing better surgical planning and surgeon training in the specific case beforehand.

\section{Authors' roles \& responsibilities}

ENG The prototypes were designed and constructed; the prototypes were tested; final approval of the version to be published

RRD Revising it critically for important intellectual content; final approval of the version to be published

BAR The prototypes were designed and constructed; final approval of the version to be published

JADS The prototypes were tested; final approval of the version to be published

FJSD The prototypes were tested; final approval of the version to be published

EKS Revising it critically for important intellectual content; final approval of the version to be published

WJG Revising it critically for important intellectual content; final approval of the version to be published

FBJ Revising it critically for important intellectual content; final approval of the version to be published

\section{REFERENCES}

1. Nienaber CA, Kische S, Rousseau H, Eggebrecht H, Rehders TC, Kundt G, et al; INSTEAD-XL trial. Endovascular repair of type B aortic dissection: long-term results of the randomized investigation of stent grafts in aortic dissection trial. Circ Cardiovasc Interv. 2013;6(4):407-16.

2. Ziza V, Canaud L, Molinari N, Branchereau P, Marty-Ané C, Alric P. Thoracic endovascular aortic repair: a single center's 15-year experience. JThorac Cardiovasc Surg. 2016;151(6):1595-603.

3. Moulakakis KG, Mylonas SN, Dalainas I, Kakisis J, Kotsis T, Liapis CD. Management of complicated and uncomplicated acute type B dissection. A systematic review and meta-analysis. Ann Cardiothorac Surg. 2014;3(3):234-46.

4. Fattori R, Montgomery D, Lovato L, Kische S, Di Eusanio M, Ince H, et al. Survival after endovascular therapy in patients with type B aortic dissection: a report from the International Registry of Acute Aortic Dissection (IRAD). JACC Cardiovasc Interv. 2013;6(8):876-82.

5. Ulug P, McCaslin JE, Stansby G, Powell JT. Endovascular versus conventional medical treatment for uncomplicated chronic type B aortic dissection. Cochrane Database Syst Rev. 2012;11:CD006512.
6. Erbel R, Aboyans V, Boileau C, Bossone E, Bartolomeo RD, Eggebrecht $\mathrm{H}$, et al; ESC Committee for Practice Guidelines. 2014 ESC Guidelines on the diagnosis and treatment of aortic diseases: Document covering acute and chronic aortic diseases of the thoracic and abdominal aorta of the adult. The Task Force for the Diagnosis and Treatment of Aortic Diseases of the European Society of Cardiology (ESC). Eur Heart J. 2014;35(41):2873-926.

7. Torres $I O$, De Luccia N. A simulator for training in endovascular aneurysm repair: the use of three dimensional printers. Eur J Vasc Endovasc Surg. 2017;54(2):247-53

8. Esses SJ, Berman P, Bloom Al, Sosna J. Clinical applications of physical 3D models derived from MDCT data and created by rapid prototyping. AJR Am J Roentgenol. 2011;196(6):W683-8.

9. Tam MD, Latham T, Brown JR, Jakeways M. Use of a 3D printed hollow aortic model to assist EVAR planning in a case with complex neck anatomy: potential of 3D printing to improve patient outcome. J Endovasc Ther. 2014;21(5):760-2.

10. Biglino G, Verschueren P, Zegels R, Taylor AM, Schievano S. Rapid prototyping compliant arterial phantoms for in-vitro studies and device testing. J Cardiovasc Magn Reson. 2013;15:2.

11. Valverde I, Gomez G, Coserria JF, Suarez-Mejias C, Uribe S, Sotelo J, et al. 3D printed models for planning endovascular stenting in transverse aortic arch hypoplasia. Catheter Cardiovasc Interv. 2015;85(6):1006-12.

12. Sodian R, Schmauss D, Schmitz C, Bigdeli A, Haeberle S, Schmoeckel $M$, et al. 3-dimensional printing of models to create custom-made devices for coil embolization of an anastomotic leak after aortic arch replacement. Ann Thorac Surg. 2009;88(3):974-8.

13. Bangeas P, Voulalas G, Ktenidis K. Rapid prototyping in aortic surgery. Interact Cardiovasc Thorac Surg. 2016;22(4):513-4.

14. Heller M, Bauer HK, Goetze E, Gielisch M, Roth KE, Drees P, et al. Applications of patient-specific 3D printing in medicine. Int J Comput Dent. 2016;19(4):323-39.

15. Mitchell RS, Ishimaru S, Ehrlich MP, Iwase T, Lauterjung L, Shimono T, et al. First International Summit on Thoracic Aortic Endografting: roundtable on thoracic aortic dissection as an indication for endografting. J Endovasc Ther. 2002;9(Suppl 2):II98-105.

16. Moulakakis KG, Mylonas SN, Markatis F, Kotsis T, Kakisis J, Liapis CD. A systematic review and meta-analysis of hybrid aortic arch replacement. Ann Cardiothorac Surg. 2013;2(3):247-60.

17. Vallabhajosyula P, Szeto WY, Desai N, Komlo C, Bavaria JE. Type II arch hybrid debranching procedure. Ann Cardiothoracic Surg. 2013;2(3):378-86.

18. Hori D, Okamura H, Yamamoto T, Nishi S, Yuri K, Kimura N, et al. Early and mid-term outcomes of endovascular and open surgical repair of non-dissected aortic arch aneurysm. Interact Cardiovasc Thorac Surg. 2017;24(6):944-50.

19. Yoshitake A, Okamoto K, Yamazaki M, Kimura N, Hirano A, lida Y, et al. Comparison of aortic arch repair using the endovascular technique, total arch replacement and staged surgery. Eur J Cardiothorac Surg. 2017:51(6):1142-8

20. Giannopoulos AA, Mitsouras D, Yoo SJ, Liu PP, Chatzizisis YS, Rybicki FJ. Applications of 3D printing in cardiovascular diseases. Nat Rev Cardiol. 2016;13(12):701-18.

21. Crafts TD, Ellsperman SE, WannemuehlerTJ, BellicchiTD, Shipchandler TZ, Mantravadi AV. Three-dimensional printing and its applications in otorhinolaryngology-head and neck surgery. Otolaryngol Head Neck Surg. 2017;156(6):999-1010.

22. Luo H, Meyer-Szary J, Wang Z, Sabiniewicz R, Liu Y. Three-dimensional printing in cardiology: current applications and future challenges. Cardiol J. 2017;24(4):436-44. 